\title{
QUANTUM STATES SATISFYING CLASSICAL PROBABILITY CONSTRAINTS
}

\author{
ELENA R. LOUBENETS \\ Applied Mathematics Department \\ Moscow State Institute of Electronics and Mathematics, Technical University \\ Trekhsvyatitelskii Per. 3/12, Moscow 109028, Russia \\ E-mail:erl@erl.msk.ru
}

\begin{abstract}
For linear combinations of quantum product averages in an arbitrary bipartite state, we derive new quantum Bell-form and $\mathrm{CHSH}$-form inequalities with the right-hand sides expressed in terms of a bipartite state. This allows us to specify bipartite state properties sufficient for the validity of a classical CHSH-form inequality and the perfect correlation form of the original Bell inequality for any bounded quantum observables. We also introduce a new general condition on a bipartite state and quantum observables sufficient for the validity of the original Bell inequality, in its perfect correlation or anticorrelation forms. Under this general sufficient condition, a bipartite quantum state does not necessarily exhibit Bell's perfect correlations or anticorrelations.
\end{abstract}

1. Introduction. The Bell [1] and the Clauser-Horne-Shimony-Holt $(\mathrm{CHSH})[2]$ inequalities, derived originally in the frame of the Bell local hidden variable model, describe the relations between the product expectation values under different joint measurements.

In the frame of classical probability, the product expectation values in every classical state satisfy the $\mathrm{CHSH}$ inequality and the perfect correlation form of the original Bell inequality $\left({ }^{1}\right)$ for any bounded classical observables.

In the frame of quantum probability, under joint quantum measurements on a bipartite system, the product expectation values in a bipartite quantum state do not, in general, satisfy the above inequalities. It is, however, well known $[4,5]$ that not only all separable quantum states but also a variety of nonseparable quantum states satisfy the CHSH inequality for any bounded quantum observables. We also proved in [3] (section 3.B.1,

2000 Mathematics Subject Classification: 81P15, 47A20, 47A63.

The paper is in final form and no version of it will be published elsewhere.

$\left({ }^{1}\right)$ The original proof [1] of the perfect correlation form of the Bell inequality is true only for dichotomic classical observables admitting values $\pm \lambda$. In appendix of [3], we proved the validity of this inequality for any three bounded classical observables. 
item 1 ) that there exist $\left({ }^{2}\right)$ separable quantum states that satisfy the perfect correlation form of the original Bell inequality for any bounded quantum observables $\left({ }^{3}\right)$.

At present, Bell-type inequalities are widely used in quantum information processing. However, from the pioneering paper of R. Werner [4] up to now a general analytical structure of bipartite quantum states satisfying a classical $\left({ }^{4}\right) \mathrm{CHSH}$-form inequality has not been well formalized. Moreover, a structure of bipartite quantum states satisfying the perfect correlation form of the original Bell inequality for any bounded quantum observables has been analyzed [3] in the literature only in the separable case.

The aim of this paper is to introduce general analytical conditions sufficient for a bipartite quantum state to satisfy a classical CHSH-form inequality and the perfect correlation form of the original Bell inequality for any bounded quantum observables.

In section 2.1, we introduce a new notion: a source-operator for a bipartite quantum state and prove (proposition 1) that, for any bipartite state, source-operators exist. We specify new notions: density source-operator (DSO) states and Bell class states, and present examples of such bipartite states. We prove (proposition 2) that the nonseparable Werner state $\left(^{5}\right)$ is a DSO state for any dimension $d \geq 2$ and represents a Bell class state if $d \geq 3$.

In sections 2.2, 2.3, for an arbitrary bipartite state, we derive (propositions 3, 4) new upper bounds of linear combinations of quantum product averages. These upper bounds are expressed in terms of source-operators for a bipartite state, and this allows us to specify analytically in section 3 the situations where a bipartite quantum state satisfies a classical Bell-type inequality.

In section 3:

(i) we prove (theorems 1,2 ) that, for any bounded quantum observables $\left({ }^{6}\right)$, the product expectation values in a density source-operator (DSO) state satisfy a classical CHSH-form inequality;

(ii) we prove (theorem 3) that every Bell class state satisfies the perfect correlation form of the original Bell inequality for any bounded quantum observables and does not necessarily exhibit perfect correlations;

(iii) we introduce (theorem 4) a new general condition sufficient for a density sourceoperator (DSO) state and three bounded quantum observables to satisfy the original Bell inequality, in its perfect correlation or anticorrelation forms. A DSO state, satisfying this general sufficient condition, does not necessarily exhibit (proposition 5) Bell's perfect correlations or anticorrelations [1].

$\left({ }^{2}\right)$ See Eq. (49) in [3].

$\left({ }^{3}\right)$ These separable quantum states do not necessarily exhibit Bell's perfect correlations [1], see the discussion in [3], section 3.B.1, item 1 .

$\left({ }^{4}\right)$ Here, the term classical specifies the validity of some probabilistic constraint in the frame of classical probability.

$\left({ }^{5}\right)$ This bipartite quantum state was introduced by R. Werner in [4] and is widely used in quantum information processing.

$\left({ }^{6}\right)$ Everywhere in this paper, quantum observables may be of any spectral types. 
In section 4, we specify (theorems 5-7) the validity of classical Bell-type inequalities under generalized quantum measurements of Alice and Bob.

2. Quantum upper bounds. General case. Let a bipartite quantum system be described in terms of a separable complex Hilbert space $\mathcal{H}_{1} \otimes \mathcal{H}_{2}$. In this section, for an arbitrary state $\left({ }^{7}\right) \rho$ on $\mathcal{H}_{1} \otimes \mathcal{H}_{2}$, we derive new upper bounds of linear combinations of quantum product averages:

$$
\begin{aligned}
& \operatorname{tr}\left[\rho\left(W_{1}^{(a)} \otimes W_{2}^{\left(b_{1}\right)}\right)\right]-\operatorname{tr}\left[\rho\left(W_{1}^{(a)} \otimes W_{2}^{\left(b_{2}\right)}\right)\right], \\
& \operatorname{tr}\left[\rho\left(W_{1}^{\left(a_{1}\right)} \otimes W_{2}^{(b)}\right)\right]-\operatorname{tr}\left[\rho\left(W_{1}^{\left(a_{2}\right)} \otimes W_{2}^{(b)}\right)\right], \\
& \sum_{n, m=1,2} \gamma_{n m} \operatorname{tr}\left[\rho\left(W_{1}^{\left(a_{n}\right)} \otimes W_{2}^{\left(b_{m}\right)}\right)\right] .
\end{aligned}
$$

Here, $W_{1}^{(a)}, W_{2}^{(b)}$ are any bounded quantum observables on $\mathcal{H}_{1}$ and $\mathcal{H}_{2}$, respectively, and $\gamma_{n m}, n, m=1,2$, are any real coefficients. For clarity, we label $\left(^{8}\right)$ by indices " $a$ " quantum observables on $\mathcal{H}_{1}$ and by "b" on $\mathcal{H}_{2}$.

2.1. Source-operators for a bipartite state. In order to evaluate (1) and (2), we introduce in a general setting a new notion.

Denote by $\mathcal{K}_{112}:=\mathcal{H}_{1} \otimes \mathcal{H}_{1} \otimes \mathcal{H}_{2}$ and $\mathcal{K}_{122}:=\mathcal{H}_{1} \otimes \mathcal{H}_{2} \otimes \mathcal{H}_{2}$ the extended tensor product Hilbert spaces. Below, we use the notation $\operatorname{tr}_{\mathcal{H}_{m}}^{(k)}[\cdot], k=1,2,3, m=1,2$, for the partial trace over the elements of a Hilbert space $\mathcal{H}_{m}$ standing in the $k$-th place of tensor products.

Definition 1. For a state $\rho$ on $\mathcal{H}_{1} \otimes \mathcal{H}_{2}$, let $T_{112}$ on $\mathcal{K}_{112}$ and $T_{122}$ on $\mathcal{K}_{122}$ be self-adjoint trace class operators defined by the relations:

$$
\begin{array}{ll}
\operatorname{tr}_{\mathcal{H}_{1}}^{(1)}\left[T_{112}\right]=\rho, & \operatorname{tr}_{\mathcal{H}_{1}}^{(2)}\left[T_{112}\right]=\rho ; \\
\operatorname{tr}_{\mathcal{H}_{2}}^{(2)}\left[T_{122}\right]=\rho, & \operatorname{tr}_{\mathcal{H}_{2}}^{(3)}\left[T_{122}\right]=\rho .
\end{array}
$$

We call any of these dilations a source-operator for a bipartite state $\rho$.

Proposition 1. For a state $\rho$ on $\mathcal{H}_{1} \otimes \mathcal{H}_{2}$, there exist source-operators $T_{122}$ and $T_{112}$.

Proof. The spectral decomposition of a quantum state $\rho$ on $\mathcal{H}_{1} \otimes \mathcal{H}_{2}$ reads:

$$
\rho=\sum_{i} \alpha_{i}\left|\Psi_{i}\right\rangle\left\langle\Psi_{i}\right|, \quad\left\langle\Psi_{i}, \Psi_{j}\right\rangle=\delta_{i j}, \quad \forall \alpha_{i}>0, \quad \sum_{i} \alpha_{i}=1 .
$$

Take an orthonormal basis $\left\{\varphi_{n}\right\}$ in $\mathcal{H}_{2}$ and consider the Schmidt decomposition of an eigenvector $\Psi_{i}$ with respect to this basis:

$$
\Psi_{i}=\sum_{n} \Phi_{n}^{(i)} \otimes \varphi_{n}, \quad \sum_{n}\left\langle\Phi_{n}^{(i)}, \Phi_{n}^{(j)}\right\rangle=\delta_{i j} .
$$

Substituting (6) into (5), we derive

$$
\rho=\sum_{n, m} \rho_{n m} \otimes\left|\varphi_{n}\right\rangle\left\langle\varphi_{m}\right|, \quad \text { where } \quad \rho_{n m}:=\sum_{i} \alpha_{i}\left|\Phi_{n}^{(i)}\right\rangle\left\langle\Phi_{m}^{(i)}\right|, \quad \forall n, m .
$$

The operators $\rho_{n n}$ are positive with $\sum_{n} \operatorname{tr}\left[\rho_{n n}\right]=1$.

$\left({ }^{7}\right)$ We consider only normal quantum states.

$\left.{ }^{8}\right)$ In the physical literature, these labels correspond to "Alice" and "Bob" names. 
For any density operator $\sigma$ on $\mathcal{H}_{2}$ and any self-adjoint trace class operator $\tau_{122}$ on $\mathcal{K}_{122}$, with $\operatorname{tr}_{\mathcal{H}_{2}}^{(2)}\left[\tau_{122}\right]=\operatorname{tr}_{\mathcal{H}_{2}}^{(3)}\left[\tau_{122}\right]=0$, the operator

$$
\begin{aligned}
T_{122}= & \sum_{n, m} \rho_{n m} \otimes\left|\varphi_{n}\right\rangle\left\langle\varphi_{m}\left|\otimes \sigma+\sum_{n, m} \rho_{n m} \otimes \sigma \otimes\right| \varphi_{n}\right\rangle\left\langle\varphi_{m}\right| \\
& -\operatorname{tr}_{\mathcal{H}_{2}}[\rho] \otimes \sigma \otimes \sigma+\tau_{122}
\end{aligned}
$$

represents a source-operator for the state (5). Here, $\operatorname{tr}_{\mathcal{H}_{2}}[\rho]=\sum_{n} \rho_{n n}$ is the density operator on $\mathcal{H}_{1}$ reduced from $\rho$. The existence of a source-operator $T_{112}$ is proved similarly.

Consider now the main properties of source-operators:

1. $\operatorname{tr}[T]=1$, for any source-operator $T$.

2. As any self-adjoint trace class operator, a source-operator admits the decomposition $T=T^{(+)}-T^{(-)}$via non-negative operators $T^{( \pm)}=\frac{1}{2}(|T| \pm T)$ and $\|T\|_{1}=$ $\operatorname{tr}\left[T^{(+)}\right]+\operatorname{tr}\left[T^{(-)}\right]$. For a source-operator, the latter relation and property 1 imply $\|T\|_{1}=1+2 \operatorname{tr}\left[T^{(-)}\right]$.

3. Any positive source-operator $T$ is a density operator and we refer to it as a density source-operator $(D S O)$. A source-operator is a DSO iff $\|T\|_{1}=1$.

DEFINITION 2. If a bipartite state has a density source-operator then we call this state a density source-operator state or a DSO state, for short.

Consider a separable state $\rho_{\text {sep }}$. Let $\sum_{m} \xi_{m} \rho_{1}^{(m)} \otimes \rho_{2}^{(m)}$, where $\xi_{m}>0, \sum_{m} \xi_{m}=1$, be a separable representation of $\rho_{\text {sep }}$. Then, for example, $T_{122}=\sum_{m} \xi_{m} \rho_{1}^{(m)} \otimes \rho_{2}^{(m)} \otimes \rho_{2}^{(m)}$ is a density source-operator for $\rho_{\text {sep }}$.

Hence, any separable state is a DSO state. However, the converse is not true and a DSO state may be nonseparable. In section 2.1.1, we consider examples of nonseparable DSO states, in particular, on an infinite dimensional Hilbert space.

If $\mathcal{H}_{1}=\mathcal{H}_{2}=\mathcal{H}$ then $\mathcal{K}_{122}=\mathcal{K}_{112}=\mathcal{H} \otimes \mathcal{H} \otimes \mathcal{H}$ and in order to distinguish between source-operators $T_{112}$ and $T_{122}$ we further label $\left({ }^{9}\right)$ them as $T_{\boldsymbol{\iota}}$ and $T_{\boldsymbol{}}$, respectively. Moreover, if there exists a source-operator that satisfies both conditions in definition 1 , then we denote it by $T \boldsymbol{\triangleleft}$. The latter source-operator has the special dilation property:

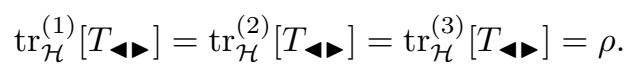

Definition 3. If, for a density source-operator (DSO) state on $\mathcal{H} \otimes \mathcal{H}$, there exists a density source-operator with the special dilation property (8) then we refer to this DSO state as a Bell class state.

The Bell class includes both separable and nonseparable states. Separable states on $\mathcal{H} \otimes \mathcal{H}$ of the special form (49) introduced in [3], namely, of the form: $\sum_{m} \xi_{m} \rho^{(m)} \otimes \rho^{(m)}$, $\xi_{m}>0, \sum_{m} \xi_{m}=1$, constitute examples of separable Bell class states.

2.1.1. Examples of DSO and Bell class states. In this section, we present examples of nonseparable DSO and Bell class states on $\mathcal{H} \otimes \mathcal{H}$.

Consider the nonseparable Werner state [4]

$$
\rho_{W}^{(d)}=\frac{d+1}{d^{3}} I_{\mathbb{C}^{d} \otimes \mathbb{C}^{d}}-\frac{1}{d^{2}} V_{d}
$$

$\left({ }^{9}\right)$ These labels indicate a "direction" of dilation. 
on $\mathbb{C}^{d} \otimes \mathbb{C}^{d}, \forall d \geq 2$. Here, $V_{d}$ is the permutation operator: $V_{d}\left(\psi_{1} \otimes \psi_{2}\right):=\psi_{2} \otimes \psi_{1}$, $\forall \psi_{1}, \psi_{2} \in \mathbb{C}^{d}$. This operator is self-adjoint and has the properties: $\left(V_{d}\right)^{2}=I_{\mathbb{C}^{d} \otimes \mathbb{C}^{d}}$, $\operatorname{tr}\left[V_{d}\right]=d$.

Proposition 2. The nonseparable Werner state $\rho_{W}^{(d)}, \forall d \geq 2$, represents a DSO state and is of the Bell class for any $d \geq 3$.

Proof. Introduce on $\mathbb{C}^{d} \otimes \mathbb{C}^{d} \otimes \mathbb{C}^{d}, \forall d \geq 3$, the orthogonal projection

(10) $\quad Q_{d}^{(-)}\left(\psi_{1} \otimes \psi_{2} \otimes \psi_{3}\right):=\frac{1}{6}\left\{\psi_{1} \otimes \psi_{2} \otimes \psi_{3}-\psi_{2} \otimes \psi_{1} \otimes \psi_{3}-\psi_{1} \otimes \psi_{3} \otimes \psi_{2}\right.$ $\left.-\psi_{3} \otimes \psi_{2} \otimes \psi_{1}+\psi_{2} \otimes \psi_{3} \otimes \psi_{1}+\psi_{3} \otimes \psi_{1} \otimes \psi_{2}\right\}$,

$\forall \psi_{1}, \psi_{2}, \psi_{3} \in \mathbb{C}^{d}$. This projection has the form:

$$
\begin{aligned}
(11) \quad 6 Q_{d}^{(-)}= & I_{\mathbb{C}^{d} \otimes \mathbb{C}^{d} \otimes \mathbb{C}^{d}}-V_{d} \otimes I_{\mathbb{C}^{d}}-I_{\mathbb{C}^{d}} \otimes V_{d}-\left(I_{\mathbb{C}^{d}} \otimes V_{d}\right)\left(V_{d} \otimes I_{\mathbb{C}^{d}}\right)\left(I_{\mathbb{C}^{d}} \otimes V_{d}\right) \\
& +\left(I_{\mathbb{C}^{d}} \otimes V_{d}\right)\left(V_{d} \otimes I_{\mathbb{C}^{d}}\right)+\left(V_{d} \otimes I_{\mathbb{C}^{d}}\right)\left(I_{\mathbb{C}^{d}} \otimes V_{d}\right)
\end{aligned}
$$

and admits a representation:

$$
\begin{aligned}
6 Q_{d}^{(-)}= & I_{\mathbb{C}^{d} \otimes \mathbb{C}^{d} \otimes \mathbb{C}^{d}}-\sum_{n, m}\left|e_{n}\right\rangle\left\langle e_{m}|\otimes| e_{m}\right\rangle\left\langle e_{n}\left|\otimes I_{\mathbb{C}^{d}}-\sum_{n, m} I_{\mathbb{C}^{d}} \otimes\right| e_{n}\right\rangle\left\langle e_{m}|\otimes| e_{m}\right\rangle\left\langle e_{n}\right| \\
& -\sum_{n, m}\left|e_{n}\right\rangle\left\langle e_{m}\left|\otimes I_{\mathbb{C}^{d}} \otimes\right| e_{m}\right\rangle\left\langle e_{n}\left|+\sum_{n, m, k}\right| e_{n}\right\rangle\left\langle e_{m}|\otimes| e_{m}\right\rangle\left\langle e_{k}|\otimes| e_{k}\right\rangle\left\langle e_{n}\right| \\
& +\sum_{n, m, k}\left|e_{m}\right\rangle\left\langle e_{n}|\otimes| e_{k}\right\rangle\left\langle e_{m}|\otimes| e_{n}\right\rangle\left\langle e_{k}\right|
\end{aligned}
$$

in an orthonormal basis $\left\{e_{n}\right\}$ in $\mathbb{C}^{d}$ (notice that $V_{d}=\sum_{n, m=1}^{d}\left|e_{n}\right\rangle\left\langle e_{m}|\otimes| e_{m}\right\rangle\left\langle e_{n}\right|$ ).

We have: $\operatorname{tr}_{\mathbb{C}^{d}}^{(j)}\left[Q_{d}^{(-)}\right]=\frac{d-2}{6}\left(I_{\mathbb{C}^{d} \otimes \mathbb{C}^{d}}-V_{d}\right), \forall j=1,2,3$. Hence, for the state $\rho_{W}^{(d)}, \forall d \geq 3$, the operator

$$
R_{\hookrightarrow \bullet}^{(d)}=\frac{1}{d^{4}} I_{\mathbb{C}^{d} \otimes \mathbb{C}^{d} \otimes \mathbb{C}^{d}}+\frac{6}{d^{2}(d-2)} Q_{d}^{(-)}
$$

represents a density source-operator with the special dilation property (8), that is: $\operatorname{tr}_{\mathbb{C}^{d}}^{(j)}\left[R_{\hookrightarrow \boldsymbol{\downarrow}}^{(d)}\right]=\rho_{W}^{(d)}, \forall j=1,2,3$. If $d=2$, then

$$
R_{\triangleright}^{(2)}=\frac{1}{4} I_{\mathbb{C}^{2} \otimes \mathbb{C}^{2} \otimes \mathbb{C}^{2}}-\frac{1}{8} V_{2} \otimes I_{\mathbb{C}^{2}}-\frac{1}{8}\left(I_{\mathbb{C}^{2}} \otimes V_{2}\right)\left(V_{2} \otimes I_{\mathbb{C}^{2}}\right)\left(I_{\mathbb{C}^{2}} \otimes V_{2}\right)
$$

is a density source-operator for $\rho_{W}^{(2)}$. The existence of the density source-operators (13) and (14) proves the statement.

Consider now examples of DSO and Bell class states on an infinite dimensional Hilbert space $\mathcal{H} \otimes \mathcal{H}$. Take the quantum states

$$
\begin{aligned}
\rho_{1}= & \frac{1}{4}\left|\psi_{1} \otimes \psi_{1}+\psi_{2} \otimes \psi_{2}\right\rangle\left\langle\psi_{1} \otimes \psi_{1}+\psi_{2} \otimes \psi_{2}\right| \\
& +\frac{1}{4}\left(\left|\psi_{1}\right\rangle\left\langle\psi_{1}|+| \psi_{2}\right\rangle\left\langle\psi_{2}\right|\right) \otimes\left|\psi_{1}\right\rangle\left\langle\psi_{1}\right|
\end{aligned}
$$


and

$$
\begin{aligned}
\rho_{2}= & \frac{1}{6}\left|\psi_{1} \otimes \psi_{1}+\psi_{2} \otimes \psi_{2}\right\rangle\left\langle\psi_{1} \otimes \psi_{1}+\psi_{2} \otimes \psi_{2}\right| \\
& +\frac{1}{6}\left(\left|\psi_{1}\right\rangle\left\langle\psi_{1}|+| \psi_{2}\right\rangle\left\langle\psi_{2}\right|\right) \otimes\left|\psi_{1}\right\rangle\left\langle\psi_{1}\right| \\
& +\frac{1}{6}\left|\psi_{1}\right\rangle\left\langle\psi_{1}\right| \otimes\left(\left|\psi_{1}\right\rangle\left\langle\psi_{1}|+| \psi_{2}\right\rangle\left\langle\psi_{2}\right|\right),
\end{aligned}
$$

where $\psi_{1}, \psi_{2}$ are any mutually orthogonal unit vectors in $\mathcal{H}$. The partial transpose $\rho_{1}^{T_{1}}$ has the negative eigenvalue $\lambda=\frac{1}{8}(1-\sqrt{5})$, corresponding to the eigenvector $\psi=c\left(\psi_{1} \otimes\right.$ $\left.\psi_{2}+\frac{1-\sqrt{5}}{2} \psi_{2} \otimes \psi_{1}\right)$. Therefore, due to the Peres separability criterion [6], the state $\rho_{1}$ is nonseparable. Nonseparability of $\rho_{2}$ is proved similarly. The operators

$$
\begin{aligned}
R_{\triangleright}= & \frac{1}{4}\left|\psi_{1} \otimes \psi_{1}+\psi_{2} \otimes \psi_{2}\right\rangle\left\langle\psi_{1} \otimes \psi_{1}+\psi_{2} \otimes \psi_{2}|\otimes| \psi_{1}\right\rangle\left\langle\psi_{1}\right| \\
& +\frac{1}{4}\left|\psi_{1} \otimes \psi_{1} \otimes \psi_{1}+\psi_{2} \otimes \psi_{1} \otimes \psi_{2}\right\rangle\left\langle\psi_{1} \otimes \psi_{1} \otimes \psi_{1}+\psi_{2} \otimes \psi_{1} \otimes \psi_{2}\right|
\end{aligned}
$$

and

$$
\begin{aligned}
R_{\hookrightarrow \boldsymbol{⿰}}= & \frac{1}{6}\left|\psi_{1} \otimes \psi_{1}+\psi_{2} \otimes \psi_{2}\right\rangle\left\langle\psi_{1} \otimes \psi_{1}+\psi_{2} \otimes \psi_{2}|\otimes| \psi_{1}\right\rangle\left\langle\psi_{1}\right| \\
& +\frac{1}{6}\left|\psi_{1} \otimes \psi_{1} \otimes \psi_{1}+\psi_{2} \otimes \psi_{1} \otimes \psi_{2}\right\rangle\left\langle\psi_{1} \otimes \psi_{1} \otimes \psi_{1}+\psi_{2} \otimes \psi_{1} \otimes \psi_{2}\right| \\
& +\frac{1}{6}\left|\psi_{1} \otimes \psi_{1} \otimes \psi_{1}+\psi_{1} \otimes \psi_{2} \otimes \psi_{2}\right\rangle\left\langle\psi_{1} \otimes \psi_{1} \otimes \psi_{1}+\psi_{1} \otimes \psi_{2} \otimes \psi_{2}\right|
\end{aligned}
$$

represent density source-operators for $\rho_{1}$ and $\rho_{2}$, respectively. Moreover, the DSO (18) has the special dilation property (8). Hence: (i) the state $\rho_{1}$ is a nonseparable DSO state; (ii) the state $\rho_{2}$ is a nonseparable Bell class state.

2.2. Quantum Bell-form inequalities. Based on the new notion of a source-operator introduced in section 2.1, consider now upper bounds of linear combinations (1) of quantum product averages in an arbitrary state $\rho$ on $\mathcal{H}_{1} \otimes \mathcal{H}_{2}$.

Let $T_{122}$ and $T_{112}$ be any source-operators for a state $\rho$. According to proposition 1 , for any bipartite state $\rho$, these operators exist. In view of definition 1 , we have:

$$
\begin{aligned}
& \operatorname{tr}\left[\rho\left(W_{1}^{(a)} \otimes W_{2}^{\left(b_{1}\right)}-W_{1}^{(a)} \otimes W_{2}^{\left(b_{2}\right)}\right)\right] \\
= & \operatorname{tr}\left[T_{122}\left(W_{1}^{(a)} \otimes W_{2}^{\left(b_{1}\right)} \otimes I_{\mathcal{H}_{2}}-W_{1}^{(a)} \otimes I_{\mathcal{H}_{2}} \otimes W_{2}^{\left(b_{2}\right)}\right)\right], \\
& \operatorname{tr}\left[\rho\left(W_{1}^{\left(a_{1}\right)} \otimes W_{2}^{(b)}-W_{1}^{\left(a_{2}\right)} \otimes W_{2}^{(b)}\right)\right] \\
= & \operatorname{tr}\left[T_{112}\left(W_{1}^{\left(a_{1}\right)} \otimes I_{\mathcal{H}_{1}} \otimes W_{2}^{(b)}-I_{\mathcal{H}_{1}} \otimes W_{1}^{\left(a_{2}\right)} \otimes W_{2}^{(b)}\right)\right],
\end{aligned}
$$

and these representations allow us to prove the following general statement.

Proposition 3. Let $W_{1}^{(a)}$ and $W_{2}^{(b)}$ be any bounded quantum observables on $\mathcal{H}_{1}$ and $\mathcal{H}_{2}$, respectively, with operator norms $\|\cdot\| \leq 1$. An arbitrary state $\rho$ on $\mathcal{H}_{1} \otimes \mathcal{H}_{2}$ satisfies the inequalities

$$
\begin{aligned}
& \left|\operatorname{tr}\left[\rho\left(W_{1}^{(a)} \otimes W_{2}^{\left(b_{1}\right)}\right)\right]-\operatorname{tr}\left[\rho\left(W_{1}^{(a)} \otimes W_{2}^{\left(b_{2}\right)}\right)\right]\right| \\
& \quad \leq\left\|T_{122}\right\|_{1}\left\{1-\operatorname{tr}\left[\sigma_{T_{122}}\left(W_{2}^{\left(b_{1}\right)} \otimes W_{2}^{\left(b_{2}\right)}\right)\right]\right\}
\end{aligned}
$$


and

$$
\begin{aligned}
& \left|\operatorname{tr}\left[\rho\left(W_{1}^{\left(a_{1}\right)} \otimes W_{2}^{(b)}\right)\right]-\operatorname{tr}\left[\rho\left(W_{1}^{\left(a_{2}\right)} \otimes W_{2}^{(b)}\right)\right]\right| \\
& \quad \leq\left\|T_{112}\right\|_{1}\left\{1-\operatorname{tr}\left[\sigma_{T_{112}}\left(W_{1}^{\left(a_{1}\right)} \otimes W_{1}^{\left(a_{2}\right)}\right)\right]\right\}
\end{aligned}
$$

where $T_{122}$ and $T_{112}$ are any source-operators for $\rho$ and

$$
\sigma_{T_{122}}:=\frac{1}{\left\|T_{122}\right\|_{1}} \operatorname{tr}_{\mathcal{H}_{1}}^{(1)}\left[\left|T_{122}\right|\right], \quad \sigma_{T_{112}}:=\frac{1}{\left\|T_{112}\right\|_{1}} \operatorname{tr}_{\mathcal{H}_{2}}^{(3)}\left[\left|T_{112}\right|\right]
$$

are density operators on $\mathcal{H}_{2} \otimes \mathcal{H}_{2}$ and $\mathcal{H}_{1} \otimes \mathcal{H}_{1}$, respectively.

In the right-hand side of (20) (or (21)), the observables can be interchanged.

Proof. In order to prove (20), we notice that in (19):

$$
\begin{aligned}
& W_{1}^{(a)} \otimes W_{2}^{\left(b_{1}\right)} \otimes I_{\mathcal{H}_{2}}=\left(W_{1}^{(a)} \otimes I_{\mathcal{H}_{2}} \otimes I_{\mathcal{H}_{2}}\right)\left(I_{\mathcal{H}_{1}} \otimes W_{2}^{\left(b_{1}\right)} \otimes I_{\mathcal{H}_{2}}\right), \\
& W_{1}^{(a)} \otimes I_{\mathcal{H}_{2}} \otimes W_{2}^{\left(b_{2}\right)}=\left(W_{1}^{(a)} \otimes I_{\mathcal{H}_{2}} \otimes I_{\mathcal{H}_{2}}\right)\left(I_{\mathcal{H}_{1}} \otimes I_{\mathcal{H}_{2}} \otimes W_{2}^{\left(b_{2}\right)}\right),
\end{aligned}
$$

and the bounded quantum observables

$$
W_{1}^{(a)} \otimes I_{\mathcal{H}_{2}} \otimes I_{\mathcal{H}_{2}}, \quad I_{\mathcal{H}_{1}} \otimes W_{2}^{\left(b_{1}\right)} \otimes I_{\mathcal{H}_{2}}, \quad I_{\mathcal{H}_{1}} \otimes I_{\mathcal{H}_{2}} \otimes W_{2}^{\left(b_{2}\right)}
$$

on $\mathcal{K}_{122}$ mutually commute. From the von Neumann theorem ([7], page 221) it follows that there exist:

(i) a bounded quantum observable $V_{a}^{\left(b_{1}, b_{2}\right)}$ on $\mathcal{K}_{122}$;

(ii) bounded Borel real-valued functions $\varphi_{1}^{(a)}, \varphi_{2}^{\left(b_{1}\right)}, \varphi_{3}^{\left(b_{2}\right)}$ on $\left(\mathbb{R}, \mathcal{B}_{\mathbb{R}}\right)$, with supremum norms $\left\|\varphi_{1}^{(a)}\right\|,\left\|\varphi_{2}^{\left(b_{1}\right)}\right\|,\left\|\varphi_{3}^{\left(b_{2}\right)}\right\| \leq 1$

such that

$$
\begin{aligned}
& W_{1}^{(a)} \otimes I_{\mathcal{H}_{2}} \otimes I_{\mathcal{H}_{2}}=\varphi_{1}^{(a)}\left(V_{a}^{\left(b_{1}, b_{2}\right)}\right), \quad I_{\mathcal{H}_{1}} \otimes W_{2}^{\left(b_{1}\right)} \otimes I_{\mathcal{H}_{2}}=\varphi_{2}^{\left(b_{1}\right)}\left(V_{a}^{\left(b_{1}, b_{2}\right)}\right), \\
& I_{\mathcal{H}_{1}} \otimes I_{\mathcal{H}_{2}} \otimes W_{2}^{\left(b_{2}\right)}=\varphi_{3}^{\left(b_{2}\right)}\left(V_{a}^{\left(b_{1}, b_{2}\right)}\right) .
\end{aligned}
$$

Let $P_{V_{a}^{\left(b_{1}, b_{2}\right)}}(\cdot)$, where $P_{V_{a}^{\left(b_{1}, b_{2}\right)}}(\mathbb{R})=I_{\mathcal{K}_{122}}$, be the projection-valued measure corresponding uniquely to $V_{a}^{\left(b_{1}, b_{2}\right)}$ due to the spectral theorem. In view of (19) and (25),

$$
\begin{aligned}
& \operatorname{tr}\left[\rho\left(W_{1}^{(a)} \otimes W_{2}^{\left(b_{1}\right)}\right)\right]=\int_{\mathbb{R}} \varphi_{1}^{(a)}(\xi) \varphi_{2}^{\left(b_{1}\right)}(\xi) \nu_{a}^{\left(b_{1}, b_{2}\right)}\left(d \xi ; T_{122}\right), \\
& \operatorname{tr}\left[\rho\left(W_{1}^{(a)} \otimes W_{2}^{\left(b_{2}\right)}\right)\right]=\int_{\mathbb{R}} \varphi_{1}^{(a)}(\xi) \varphi_{3}^{\left(b_{2}\right)}(\xi) \nu_{a}^{\left(b_{1}, b_{2}\right)}\left(d \xi ; T_{122}\right),
\end{aligned}
$$

where we denote by $\nu_{a}^{\left(b_{1}, b_{2}\right)}(\cdot ; Y)$ a $\sigma$-additive bounded real-valued measure on $\left(\mathbb{R}, \mathcal{B}_{\mathbb{R}}\right)$, defined by the relation

$$
\nu_{a}^{\left(b_{1}, b_{2}\right)}(\cdot ; Y):=\operatorname{tr}\left[Y P_{V_{a}^{\left(b_{1}, b_{2}\right)}}(\cdot)\right], \quad \nu_{a}^{\left(b_{1}, b_{2}\right)}(\mathbb{R} ; Y)=\operatorname{tr}[Y],
$$

for any self-adjoint trace class operator $Y$ on $\mathcal{K}_{122}$. For a source-operator $T_{122}$, the measure $\nu_{a}^{\left(b_{1}, b_{2}\right)}\left(\cdot ; T_{122}\right)$ is normalized but not, in general, positive. Due to property 2 , section 2.1 ,

$$
\nu_{a}^{\left(b_{1}, b_{2}\right)}\left(\cdot ; T_{122}\right)=\nu_{a}^{\left(b_{1}, b_{2}\right)}\left(\cdot ; T_{122}^{(+)}\right)-\nu_{a}^{\left(b_{1}, b_{2}\right)}\left(\cdot ; T_{122}^{(-)}\right),
$$

where $\nu_{a}^{\left(b_{1}, b_{2}\right)}\left(\cdot ; T_{122}^{( \pm)}\right)$are unnormalized positive measures with

$$
\nu_{a}^{\left(b_{1}, b_{2}\right)}\left(\mathbb{R} ; T_{122}^{(+)}\right)+\nu_{a}^{\left(b_{1}, b_{2}\right)}\left(\mathbb{R} ; T_{122}^{(-)}\right)=\nu_{a}^{\left(b_{1}, b_{2}\right)}\left(\mathbb{R} ;\left|T_{122}\right|\right)=\left\|T_{122}\right\|_{1} .
$$


Using (26), (28), the bound ||$\varphi_{1}^{(a)} \| \leq 1$, and the inequality $|x-y| \leq 1-x y$, valid for any real numbers $|x| \leq 1,|y| \leq 1$, we derive:

$$
\begin{aligned}
& \left|\operatorname{tr}\left[\rho\left(W_{1}^{(a)} \otimes W_{2}^{\left(b_{1}\right)}-W_{1}^{(a)} \otimes W_{2}^{\left(b_{2}\right)}\right)\right]\right| \\
& \quad \leq \nu_{a}^{\left(b_{1}, b_{2}\right)}\left(\mathbb{R} ;\left|T_{122}\right|\right)-\int_{\mathbb{R}} \varphi_{2}^{\left(b_{1}\right)}(\xi) \varphi_{3}^{\left(b_{2}\right)}(\xi) \nu_{a}^{\left(b_{1}, b_{2}\right)}\left(d \xi ;\left|T_{122}\right|\right) .
\end{aligned}
$$

Due to (25) and (27),

$$
\int_{\mathbb{R}} \varphi_{2}^{\left(b_{1}\right)}(\xi) \varphi_{3}^{\left(b_{2}\right)}(\xi) \nu_{a}^{\left(b_{1}, b_{2}\right)}\left(d \xi ;\left|T_{122}\right|\right)=\left\|T_{122}\right\|_{1} \operatorname{tr}\left[\sigma_{T_{122}}\left(W_{2}^{\left(b_{1}\right)} \otimes W_{2}^{\left(b_{2}\right)}\right)\right]
$$

where $\sigma_{T_{122}}:=\frac{\operatorname{tr}_{\mathcal{H}_{1}}^{(1)}\left[\left|T_{122}\right|\right]}{\left\|T_{122}\right\|_{1}}$ is a density operator on $\mathcal{H}_{2} \otimes \mathcal{H}_{2}$. Substituting (29) and (31) into (30), we finally have:

$$
\left|\operatorname{tr}\left[\rho\left(W_{1}^{(a)} \otimes W_{2}^{\left(b_{1}\right)}-W_{1}^{(a)} \otimes W_{2}^{\left(b_{2}\right)}\right)\right]\right| \leq\left\|T_{122}\right\|_{1}\left\{1-\operatorname{tr}\left[\sigma_{T_{122}}\left(W_{2}^{\left(b_{1}\right)} \otimes W_{2}^{\left(b_{2}\right)}\right)\right]\right\} .
$$

The derivation of the inequality (21) is quite similar.

Corollary 1. Let $W_{1}^{(a)}$ and $W_{2}^{(b)}$ be any bounded quantum observables with operator norms $\|\cdot\| \leq 1$. For any state $\rho$ on $\mathcal{H}_{1} \otimes \mathcal{H}_{2}$, the inequalities

$$
\begin{aligned}
& \left|\operatorname{tr}\left[\rho\left(W_{1}^{(a)} \otimes W_{2}^{(b)}\right)\right]\right| \leq \frac{1}{2}\left\|T_{122}\right\|_{1}\left\{1+\operatorname{tr}\left[\sigma_{T_{122}}\left(W_{2}^{(b)} \otimes W_{2}^{(b)}\right)\right]\right\}, \\
& \left|\operatorname{tr}\left[\rho\left(W_{1}^{(a)} \otimes W_{2}^{(b)}\right)\right]\right| \leq \frac{1}{2}\left\|T_{112}\right\|_{1}\left\{1+\operatorname{tr}\left[\sigma_{T_{112}}\left(W_{1}^{(a)} \otimes W_{1}^{(a)}\right)\right]\right\}
\end{aligned}
$$

hold with arbitrary source-operators $T_{122}$ and $T_{112}$ for $\rho$ in the right hand sides.

In particular, for a Bell class state $\rho$ on $\mathcal{H} \otimes \mathcal{H}$, the relations (33) imply:

$$
\begin{aligned}
\left|\operatorname{tr}\left[\rho\left(W_{1} \otimes W_{2}\right)\right]\right| & \leq \frac{1}{2}\left\{1+\operatorname{tr}\left[\rho\left(W_{2} \otimes W_{2}\right)\right]\right\}, \\
\left|\operatorname{tr}\left[\rho\left(W_{1} \otimes W_{2}\right)\right]\right| & \leq \frac{1}{2}\left\{1+\operatorname{tr}\left[\rho\left(W_{1} \otimes W_{1}\right)\right]\right\},
\end{aligned}
$$

for any $W_{1}$ and $W_{2}$ on $\mathcal{H}$.

2.3. Quantum CHSH-form inequalities. Consider now upper bounds for a linear combination (2).

Proposition 4. Let $W_{1}^{\left(a_{n}\right)}$ and $W_{2}^{\left(b_{m}\right)}, n, m=1,2$, be any bounded quantum observables with operator norms $\|\cdot\| \leq 1$ and $\gamma_{n m}, n, m=1,2$, be any real coefficients with $\left|\gamma_{n m}\right| \leq 1$.

An arbitrary quantum state $\rho$ on $\mathcal{H}_{1} \otimes \mathcal{H}_{2}$ satisfies the inequality

$$
\left|\sum_{n, m=1,2} \gamma_{n m} \operatorname{tr}\left[\rho\left(W_{1}^{\left(a_{n}\right)} \otimes W_{2}^{\left(b_{m}\right)}\right)\right]\right| \leq 2\left\|T_{122}\right\|_{1},
$$

whenever $\gamma_{11} \gamma_{12}=-\gamma_{21} \gamma_{22}$, and the inequality

$$
\left|\sum_{n, m=1,2} \gamma_{n m} \operatorname{tr}\left[\rho\left(W_{1}^{\left(a_{n}\right)} \otimes W_{2}^{\left(b_{m}\right)}\right)\right]\right| \leq 2\left\|T_{112}\right\|_{1},
$$

whenever $\gamma_{11} \gamma_{21}=-\gamma_{12} \gamma_{22}$. Here, $T_{122}$ and $T_{112}$ are any source-operators for a state $\rho$. 
Proof. Due to the upper bounds (20) and (21), we have:

$$
\begin{aligned}
& \left|\sum_{n, m=1,2} \gamma_{n m} \operatorname{tr}\left[\rho\left(W_{1}^{\left(a_{n}\right)} \otimes W_{2}^{\left(b_{m}\right)}\right)\right]\right| \\
& \leq\left\|T_{122}\right\|_{1}\left\{2+\left(\gamma_{11} \gamma_{12}+\gamma_{21} \gamma_{22}\right) \operatorname{tr}\left[\sigma_{T_{122}}\left(W_{2}^{\left(b_{1}\right)} \otimes W_{2}^{\left(b_{2}\right)}\right)\right]\right\} \\
& \left|\sum_{n, m=1,2} \gamma_{n m} \operatorname{tr}\left[\rho\left(W_{1}^{\left(a_{n}\right)} \otimes W_{2}^{\left(b_{m}\right)}\right)\right]\right| \\
& \quad \leq\left\|T_{112}\right\|_{1}\left\{2+\left(\gamma_{11} \gamma_{21}+\gamma_{12} \gamma_{22}\right) \operatorname{tr}\left[\sigma_{T_{112}}\left(W_{1}^{\left(a_{1}\right)} \otimes W_{1}^{\left(a_{2}\right)}\right)\right]\right\}
\end{aligned}
$$

and these relations prove the statement.

3. Validity of classical Bell-type inequalities in the quantum case. Propositions 3 and 4 clearly indicate the cases where a bipartite quantum state satisfies a classical CHSH-form inequality and the original Bell inequality for any bounded quantum observables. Notice that, in our setting, bounded quantum observables may be of any spectral types.

TheOREm 1 (DSO states and the CHSH inequality). A density source-operator $\left({ }^{10}\right)$ (DSO) state $\rho$ on $\mathcal{H}_{1} \otimes \mathcal{H}_{2}$ satisfies the original CHSH inequality [2]:

$$
\left|\operatorname{tr}\left[\rho\left(W_{1}^{\left(a_{1}\right)} \otimes W_{2}^{\left(b_{1}\right)}+W_{1}^{\left(a_{1}\right)} \otimes W_{2}^{\left(b_{2}\right)}+W_{1}^{\left(a_{2}\right)} \otimes W_{2}^{\left(b_{1}\right)}-W_{1}^{\left(a_{2}\right)} \otimes W_{2}^{\left(b_{2}\right)}\right)\right]\right| \leq 2,
$$

for any bounded quantum observables $W_{1}^{\left(a_{n}\right)}, W_{2}^{\left(b_{m}\right)}, n, m=1,2$, with operator norms $\|\cdot\| \leq 1$.

If a DSO state on $\mathcal{H} \otimes \mathcal{H}$ is symmetric then, for this state, density source-operators $R_{\downarrow}$ and $R_{\triangleleft}$ exist simultaneously, and from proposition 4 there follows:

TheOREM 2. Let $\gamma_{n m}, n, m=1,2$, be any real coefficients with $\left|\gamma_{n m}\right| \leq 1$ such that $\gamma_{11} \gamma_{12}=-\gamma_{21} \gamma_{22}$ or $\gamma_{11} \gamma_{21}=-\gamma_{12} \gamma_{22}$.

A symmetric DSO state $\rho$ on $\mathcal{H} \otimes \mathcal{H}$ satisfies the extended CHSH inequality [3]:

$$
\left|\sum_{n, m=1,2} \gamma_{n m} \operatorname{tr}\left[\rho\left(W_{1}^{\left(a_{n}\right)} \otimes W_{2}^{\left(b_{m}\right)}\right)\right]\right| \leq 2
$$

for any bounded quantum observables $W_{1}^{\left(a_{n}\right)}, W_{2}^{\left(b_{m}\right)}, n, m=1,2$, with operator norms $\|\cdot\| \leq 1$.

Due to proposition 3, we have the following general statement on Bell class states $\left({ }^{11}\right)$.

Theorem 3 (Bell class states and the Bell inequality). A Bell class state $\rho$ on $\mathcal{H} \otimes \mathcal{H}$ satisfies the perfect correlation form of the original Bell inequality [1]:

$\left({ }^{10}\right)$ See definition 2, section 2.1.

$\left({ }^{11}\right)$ See definition 3, section 2.1. 


$$
\begin{gathered}
\left|\operatorname{tr}\left[\rho\left(W_{1} \otimes W_{2}\right)\right]-\operatorname{tr}\left[\rho\left(W_{1} \otimes \widetilde{W}_{2}\right)\right]\right| \\
\leq 1-\operatorname{tr}\left[\rho\left(W_{2} \otimes \widetilde{W}_{2}\right)\right], \\
\left|\operatorname{tr}\left[\rho\left(W_{1} \otimes W_{2}\right)\right]-\operatorname{tr}\left[\rho\left(\widetilde{W}_{1} \otimes W_{2}\right)\right]\right| \\
\leq 1-\operatorname{tr}\left[\rho\left(W_{1} \otimes \widetilde{W}_{1}\right)\right],
\end{gathered}
$$

for any bounded quantum observables $W_{1}, \widetilde{W}_{1}, W_{2}, \widetilde{W}_{2}$ on $\mathcal{H}$ with operator norms $\|\cdot\| \leq 1$. Corollary 2. Any Bell class state $\rho$ on $\mathcal{H} \otimes \mathcal{H}$ satisfies the extended CHSH inequality (40).

In the right-hand sides of the inequalities (41), the quantum observables can be interchanged.

It is necessary to underline that, in the physical literature, the validity of the perfect correlation form of the original Bell inequality for a bipartite state on $\mathcal{H} \otimes \mathcal{H}$ has been always linked with Bell's assumption of perfect correlations if the same quantum observable is measured on both sides (cf. in [1]).

In [3] (section 3.B.1, item 1) we proved that separable states of the special form $\left({ }^{12}\right)$ (49) in [3] satisfy (41) for any bounded quantum observables and do not necessarily exhibit perfect correlations. Theorem 3 generalizes this result of [3] and indicates that there exists a whole class of bipartite states, separable and nonseparable, where each state satisfies the perfect correlation form of the original Bell inequality for any three bounded quantum observables and does not necessarily exhibit perfect correlations.

In case of, for example, a dichotomic observable $W_{2}$, with eigenvalues \pm 1 , the latter means that a Bell class state $\rho$ satisfies (41) even if the correlation function $\operatorname{tr}\left[\rho\left(W_{2} \otimes\right.\right.$ $\left.\left.W_{2}\right)\right] \neq 1$.

Due to theorem 3 and proposition 2 , the nonseparable Werner state (9) on $\mathbb{C}^{d} \otimes \mathbb{C}^{d}$, $\forall d \geq 3$, satisfies the perfect correlation form of the original Bell inequality for any bounded quantum observables and does not necessarily exhibit perfect correlations.

The upper bounds in proposition 3 allow us to introduce also a condition sufficient for the validity of the original Bell inequality for a bipartite state and some three quantum observables.

THEOREM 4 (General sufficient condition). If, for a DSO state $\rho$ on $\mathcal{H} \otimes \mathcal{H}$, there exists a density source-operator $R_{\triangleright}$ such that

$$
\operatorname{tr}\left[\sigma_{R_{\triangleright}}\left(W_{2} \otimes \widetilde{W}_{2}\right)\right]= \pm \operatorname{tr}\left[\rho\left(W_{2} \otimes \widetilde{W}_{2}\right)\right], \quad \sigma_{R_{\triangleright}}=\operatorname{tr}_{\mathcal{H}}^{(1)}\left[R_{\triangleright}\right],
$$

for bounded quantum observables $W_{2}, \widetilde{W}_{2}$ on $\mathcal{H}$ with operator norms $\|\cdot\| \leq 1$ then this DSO state $\rho$ and these quantum observables $W_{2}, \widetilde{W}_{2}$ satisfy the original Bell inequality [1]:

$$
\left|\operatorname{tr}\left[\rho\left(W_{1} \otimes W_{2}\right)\right]-\operatorname{tr}\left[\rho\left(W_{1} \otimes \widetilde{W}_{2}\right)\right]\right| \leq 1 \mp \operatorname{tr}\left[\rho\left(W_{2} \otimes \widetilde{W}_{2}\right)\right],
$$

in its perfect correlation or anticorrelation form (minus or plus sign in (43), respectively). Here, $W_{1}$ is any bounded quantum observable on $\mathcal{H}$ with $\left\|W_{1}\right\| \leq 1$.

$\left({ }^{12}\right)$ As we discussed in section 2.1, these separable states belong to the Bell class. 
Notice that, in theorem 3, the sufficient condition concerns only a bipartite state property and refers only to the perfect correlation form of the Bell inequality. A Bell class state satisfies the plus sign condition (42) for any quantum observables $W_{2}, \widetilde{W}_{2}$.

In theorem 4, the sufficient condition (42) establishes the restriction on the combination: quantum observables and a DSO state, and concerns both forms of the original Bell inequality. In general, a DSO state satisfying the condition (42) does not necessarily either belong to the Bell class or satisfy (42) for any $W_{2}, \widetilde{W}_{2}$.

For a symmetric DSO state, let us now prove that the sufficient condition (42) is more general than Bell's perfect correlation/anticorrelation restriction (44) and includes the latter only as a particular case.

Proposition 5. If a symmetric DSO state $\rho$ on $\mathcal{H} \otimes \mathcal{H}$ satisfies the Bell perfect correlation or anticorrelation restriction

$$
\operatorname{tr}\left[\rho\left(W_{2} \otimes W_{2}\right)\right]= \pm 1
$$

then this DSO state satisfies the sufficient condition (42). The converse is not true.

Proof. If a DSO state $\rho$ on $\mathcal{H} \otimes \mathcal{H}$ is symmetric then it has both density source-operators, $R_{\triangleleft}$ and $R_{\downarrow}$. We have:

$$
\begin{aligned}
\operatorname{tr}\left[\sigma_{R_{\triangleright}}\left(W_{2} \otimes \widetilde{W}_{2}\right)\right] & =\operatorname{tr}\left[R_{\triangleright}\left(I_{\mathcal{H}} \otimes W_{2} \otimes \widetilde{W}_{2}\right)\right] \\
\operatorname{tr}\left[\rho\left(W_{2} \otimes \widetilde{W}_{2}\right)\right] & =\operatorname{tr}\left[R_{\triangleright}\left(W_{2} \otimes I_{\mathcal{H}} \otimes \widetilde{W}_{2}\right)\right], \\
\operatorname{tr}\left[\rho\left(W_{2} \otimes W_{2}\right)\right] & =\operatorname{tr}\left[R_{\triangleright}\left(W_{2} \otimes W_{2} \otimes I_{\mathcal{H}}\right)\right] .
\end{aligned}
$$

Using the arguments based on the von Neumann theorem [7] and quite similar to those in proposition 3, we derive:

$$
\begin{aligned}
\operatorname{tr}\left[\sigma_{R_{\triangleright}}\left(W_{2} \otimes \widetilde{W}_{2}\right)\right] & =\int_{\mathbb{R}} \varphi_{2}(\xi) \varphi_{3}(\xi) \nu\left(d \xi ; R_{\triangleright}\right), \\
\operatorname{tr}\left[\rho\left(W_{2} \otimes \widetilde{W}_{2}\right)\right] & =\int_{\mathbb{R}} \varphi_{1}(\xi) \varphi_{3}(\xi) \nu\left(d \xi ; R_{\triangleright}\right), \\
\operatorname{tr}\left[\rho\left(W_{2} \otimes W_{2}\right)\right] & =\int_{\mathbb{R}} \varphi_{1}(\xi) \varphi_{2}(\xi) \nu\left(d \xi ; R_{\triangleright}\right),
\end{aligned}
$$

where:

(i) $\nu\left(\cdot ; R_{\boldsymbol{}}\right):=\operatorname{tr}\left[R_{\triangleright} P_{V}(\cdot)\right]$ is a probability distribution on $\left(\mathbb{R}, \mathcal{B}_{\mathbb{R}}\right)$, induced $\left({ }^{(13))}\right.$ by the projection-valued measure $P_{V}$ of a quantum observable $V$ on $\mathcal{H} \otimes \mathcal{H} \otimes \mathcal{H}$ (corresponding, due to the von Neumann theorem, to three mutually commuting observables $\left.W_{2} \otimes I_{\mathcal{H}} \otimes I_{\mathcal{H}}, I_{\mathcal{H}} \otimes W_{2} \otimes I_{\mathcal{H}}, I_{\mathcal{H}} \otimes I_{\mathcal{H}} \otimes \widetilde{W}_{2}\right)$

(ii) $\varphi_{1}, \varphi_{2}, \varphi_{3}$ are bounded Borel real-valued functions on $\left(\mathbb{R}, \mathcal{B}_{\mathbb{R}}\right)$, with supremum norms $\left\|\varphi_{n}\right\| \leq 1$, such that $\varphi_{1}(V)=W_{2} \otimes I_{\mathcal{H}} \otimes I_{\mathcal{H}}, \varphi_{2}(V)=I_{\mathcal{H}} \otimes W_{2} \otimes I_{\mathcal{H}}$ and $\varphi_{3}(V)=I_{\mathcal{H}} \otimes I_{\mathcal{H}} \otimes \widetilde{W}_{2}$.

If $\rho$ satisfies the Bell restriction (44) then, due to (46),

$$
\int_{\mathbb{R}} \varphi_{1}(\xi) \varphi_{2}(\xi) \nu\left(d \xi ; R_{\triangleright}\right)= \pm 1
$$

$\left({ }^{13}\right)$ See proposition 3 . 
The latter implies $\varphi_{1}(\xi) \varphi_{2}(\xi)= \pm 1, \nu$-a.e. Since $\left\|\varphi_{1}\right\|,\left\|\varphi_{2}\right\| \leq 1$, we have $\varphi_{1}(\xi)=$ $\pm \varphi_{2}(\xi), \nu$-a.e., and, hence,

(48) $\operatorname{tr}\left[\sigma_{R_{\triangleright}}\left(W_{2} \otimes \widetilde{W}_{2}\right)\right] \mp \operatorname{tr}\left[\rho\left(W_{2} \otimes \widetilde{W}_{2}\right)\right]=\int_{\mathbb{R}}\left\{\varphi_{2}(\xi) \mp \varphi_{1}(\xi)\right\} \varphi_{3}(\xi) \nu\left(d \xi ; R_{\triangleright}\right)=0$.

The converse statement is not true and a DSO state, satisfying (48), does not necessarily satisfy (47).

Thus, a DSO state, satisfying the general sufficient condition (42) and, therefore, the original Bell inequality (43), does not necessarily exhibit Bell's perfect correlations or anticorrelations.

4. Generalized quantum measurements of Alice and Bob. In the physical literature, joint measurements on a bipartite system are usually referred to as measurements of Alice and Bob. Theorems 1-4 and proposition 5 specify the relations between the product expectation values under projective quantum measurements of Alice and Bob.

To analyze the situation under generalized joint quantum measurements on a bipartite quantum state, let us recall that an Alice/Bob joint generalized quantum measurement, with real-valued outcomes $\lambda_{1} \in \Lambda_{1}, \lambda_{2} \in \Lambda_{2}$ of any type, is described by the positive operator-valued $(P O V)$ measure

$$
M^{(a, b)}\left(B_{1} \times B_{2}\right)=M_{1}^{(a)}\left(B_{1}\right) \otimes M_{2}^{(b)}\left(B_{2}\right), \quad \forall B_{1} \subseteq \Lambda_{1}, \forall B_{2} \subseteq \Lambda_{2},
$$

where " $a$ " and $\Lambda_{1}$ refer to a setting and an outcome set on the side of Alice while " $b$ " and $\Lambda_{2}$ on the side of Bob. For simplicity, we further suppose $\left|\lambda_{1}\right| \leq 1,\left|\lambda_{2}\right| \leq 1$.

For a quantum state $\rho$ on $\mathcal{H}_{1} \otimes \mathcal{H}_{2}$, the formula $\left({ }^{14}\right)$

$$
\begin{aligned}
\left\langle\lambda_{1} \lambda_{2}\right\rangle_{\rho}^{(a, b)} & :=\int_{\Lambda_{1} \times \Lambda_{2}} \lambda_{1} \lambda_{2} \operatorname{tr}\left[\rho\left(M_{1}^{(a)}\left(d \lambda_{1}\right) \otimes M_{2}^{(b)}\left(d \lambda_{2}\right)\right)\right] \\
& =\operatorname{tr}\left[\rho\left(W_{1}^{(a)} \otimes W_{2}^{(b)}\right)\right]
\end{aligned}
$$

represents the expectation value of the product $\lambda_{1} \lambda_{2}$ of outcomes observed by Alice and Bob. Here,

$$
W_{1}^{(a)}:=\int_{\Lambda_{1}} \lambda_{1} M_{1}^{(a)}\left(d \lambda_{1}\right), \quad W_{2}^{(b)}:=\int_{\Lambda_{2}} \lambda_{2} M_{2}^{(b)}\left(d \lambda_{2}\right)
$$

are bounded quantum observables with operator norms $\|\cdot\| \leq 1$ on $\mathcal{H}_{1}$ and $\mathcal{H}_{2}$, respectively.

Theorems 1-3 and the representation (50) imply:

THEOREM 5. The product expectation values in a DSO state $\rho$ on $\mathcal{H}_{1} \otimes \mathcal{H}_{2}$ satisfy the original $\mathrm{CHSH}$ inequality:

$$
\left|\left\langle\lambda_{1} \lambda_{2}\right\rangle_{\rho}^{\left(a_{1}, b_{1}\right)}+\left\langle\lambda_{1} \lambda_{2}\right\rangle_{\rho}^{\left(a_{1}, b_{2}\right)}+\left\langle\lambda_{1} \lambda_{2}\right\rangle_{\rho}^{\left(a_{2}, b_{1}\right)}-\left\langle\lambda_{1} \lambda_{2}\right\rangle_{\rho}^{\left(a_{2}, b_{2}\right)}\right| \leq 2,
$$

under any generalized quantum measurements (49) of Alice and Bob with outcomes $\left|\lambda_{1}\right| \leq$ $1,\left|\lambda_{2}\right| \leq 1$ of any type.

$\left({ }^{14}\right)$ See also [3], section 3.A. 
THEOREM 6. Let $\gamma_{n m}, n, m=1,2$, be any real coefficients with $\left|\gamma_{n m}\right| \leq 1$ and $\gamma_{11} \gamma_{12}=$ $-\gamma_{21} \gamma_{22}$ or $\gamma_{11} \gamma_{21}=-\gamma_{12} \gamma_{22}$. If a DSO state $\rho$ on $\mathcal{H} \otimes \mathcal{H}$ is either symmetric or of the Bell class then the product expectation values in this $\rho$ satisfy the extended $C H S H$ inequality:

$$
\left|\sum_{n, m=1,2} \gamma_{n m}\left\langle\lambda_{1} \lambda_{2}\right\rangle_{\rho}^{\left(a_{n}, b_{m}\right)}\right| \leq 2,
$$

under any generalized quantum measurements (49) of Alice and Bob with outcomes $\left|\lambda_{1}\right| \leq$ $1,\left|\lambda_{2}\right| \leq 1$ of any type.

THEOREM 7. If, under joint generalized quantum measurements (49) of Alice and Bob,

$$
\int_{\Lambda_{1}} \lambda_{1} M_{1}^{\left(b_{1}\right)}\left(d \lambda_{1}\right)=\int_{\Lambda_{2}} \lambda_{2} M_{2}^{\left(b_{1}\right)}\left(d \lambda_{2}\right)
$$

then, for any Bell class state $\rho$ on $\mathcal{H} \otimes \mathcal{H}$, the product expectation values satisfy the perfect correlation form of the original Bell inequality:

$$
\left|\left\langle\lambda_{1} \lambda_{2}\right\rangle_{\rho}^{\left(a, b_{1}\right)}-\left\langle\lambda_{1} \lambda_{2}\right\rangle_{\rho}^{\left(a, b_{2}\right)}\right| \leq 1-\left\langle\lambda_{1} \lambda_{2}\right\rangle_{\rho}^{\left(b_{1}, b_{2}\right)} .
$$

The operator relation (54) does not necessarily imply the perfect correlations of outcomes on the sides of Alice and Bob and is always true in case of projective Alice and Bob measurements of the same quantum observable on both sides.

Theorem 4 can be also easily generalized to the case of joint generalized quantum measurements of Alice and Bob.

Acknowledgments. I am grateful to Marek Bożejko, Klaus Molmer and Asher Peres for valuable discussions.

\section{References}

[1] J. S. Bell, Speakable and Unspeakable in Quantum Mechanics, Cambridge University Press, Cambridge, 1987.

[2] J. F. Clauser, M. A. Horne, A. Shimony, and R. A. Holt, Phys. Rev. Lett. 23 (1969), 880-884.

[3] E. R. Loubenets, Phys. Rev. A 69 (2004), 042102.

[4] R. F. Werner, Phys. Rev. A 40 (1989), 4277.

[5] R. F. Werner and M. M. Wolf, Quantum Information and Computation 1 (2001), 1.

[6] A. Peres, Phys. Rev. Lett. 77 (1996), 1413.

[7] J. von Neumann, Mathematische Grundlagen der Quantenmechanik, Springer-Verlag, Berlin, 1932. English translation: Mathematical Foundations of Quantum Mechanics, Dover, New York, 1954. 
